\title{
Injury to Liver without mention of Open Wound into Cavity
}

National Cancer Institute

\section{Source}

National Cancer Institute. Injury to Liver without mention of Open Wound into Cavity. NCI Thesaurus. Code C35242.

Trauma to the liver without indication of associated damage to the skin and other internal and external tissues. 